\title{
Comparison of Acute and Long-term Outcomes of Evolution( and TightRail Mechanical Dilator Sheaths during Transvenous Lead Extraction
}

\author{
Nihan Bahadir ${ }^{1}$, Ugur Canpolat ${ }^{1}$, Ergün Kaya ${ }^{1}$, Levent Şahiner ${ }^{1}$, Hakan Ates ${ }^{2}$, Hikmet \\ Yorgun $^{1}$, and Kudret Aytemir ${ }^{3}$ \\ ${ }^{1}$ Hacettepe University Faculty of Medicine \\ ${ }^{2}$ Hacettepe University \\ ${ }^{3}$ Hacettepe University Medical Faculty
}

June 22, 2020

\begin{abstract}
Background: Powered transvenous lead extraction (TLE) tools are commonly required in the removal of the leads that have long implant duration due to fibrotic adhesions. However, the comparative data are lacking among different types of TLE tools. Aim: To compare the efficacy and safety of two different rotational mechanical dilator sheaths in retrospectively analyzed patients who underwent TLE. Methods and results: A total of 566 lead extractions from 302 patients using TightRailTM (333 lead extractions from 169 patients) and Evolution@ (233 lead extractions from 133 patients) mechanical dilator sheaths were performed between July 2009 and June 2018. Acute and long term outcomes of study groups were compared. There is no statistically significant difference between Evolution@ and TightRailTM groups in procedural success (93.9\% vs. 94\%), clinical success $(99.2 \%$ vs. $98 \%)$ and major complications (3.8\% vs. $1.2 \%)$, respectively ( $>>0.05)$. In multivariate regression analysis, lead dwell time, number of extracted leads, and baseline leukocyte count were found as independent predictors of procedural success $(\mathrm{p}<0.05)$. During the median follow-up of $36.6(0.2-118)$ months, all-cause mortality was observed in 73 patients $(25.6 \%$ in the Evolution@ vs. 23.1 in the TightRailTM group, p>0.05). Chronic renal disease, heart failure, and coagulopathy were shown as independent predictors of all-cause mortality in multivariate regression analysis $(\mathrm{p}<0.05)$. Conclusions: TLE using TightRailTM or Evoluation (R) mechanical dilator sheaths is a safe and effective therapeutic option. Both mechanical dilator sheaths showed similar efficacy, safety, and all-cause mortality at acute and long-term follow-up of patients who underwent TLE.
\end{abstract}

\section{Condensed Abstract}

In this observational cohort study, we compared the efficacy/safety and long-term mortality rates of the patients who underwent TLE procedure using the Evolution ${ }^{\circledR}$ vs. TightRail ${ }^{\mathrm{TM}}$ mechanical dilator sheaths. Both groups showed similar procedural success and complication rates in addition to all-cause mortality rates.

\section{What's new?}

- The Evolution ${ }^{\circledR}$ and TightRail ${ }^{\mathrm{TM}}$ Mechanical Dilator Sheaths are two different tools with variable technical properties for TLE procedure.

- To the best of our knowledge, this is the first large-scale observational report in the literature comparing the efficacy and safety of these two mechanical TLE system in chronically implanted PM/ICD leads in addition to long-term mortality outcomes. 
- Both mechanical TLE systems showed similar procedural/clinical success and complication rates and all-cause mortality at long-term follow-up.

\section{INTRODUCTION}

In parallel to the increased use of cardiac implantable electronic devices (CIED), the electrophysiology society faced with device-related problems like device upgrade, device-related infection, electrode dysfunction, and dislodgement which should be managed with effective and reliable methods ${ }^{1}$. Despite significant improvement over the last decade in transvenous lead extraction (TLE) technology, the procedure is still associated with a significant morbidity and mortality ${ }^{2}$.

After implantation, transvenous leads are often encapsulated with fibrotic capsules which adheres to vascular and intra-cardiac tissue by different humoral and cellular mechanisms ${ }^{1}$. Among various tools and methods, powered extraction devices are required in the removal of the chronically implanted leads ${ }^{3}$. Rotational mechanical dilator sheaths are acting by dissection of the fibrotic tissue in which the electrodes connected using the threaded end portion of the system ${ }^{4}$. Currently, two different mechanical dilator sheaths with variable technical properties were available in the market [Evolution ${ }^{(\mathrm{r})}$ (Cook Medical, Bloomington, IN, USA) and TightRail ${ }^{\mathrm{TM}}$ (Spectranetics Corp., Colorado Springs, Colorado, USA) mechanical dilator sheaths]. Both TLE system revealed high efficacy rates and acceptable safety results ${ }^{3,5}$. However, the comparative data are scarce among different types of powered extraction tools. Previous studies have presented the data of single TLE device in general ${ }^{5,6}$. There is only one small study regarding the comparison of different rotational mechanical dilators ${ }^{7}$.

Thus, we aimed to compare the safety and efficacy outcomes, and all-cause mortality at long-term follow-up in a large-scale study population who underwent TLE using two different rotational mechanical dilators sheaths.

\section{METHODS}

\section{Study population}

Our clinic is a high volume tertiary referral center for TLE procedure ( $>130 /$ year $)$ in our country. The study was designed as a single-center observational cohort study. The study included a total of 302 patients who underwent TLE procedure in our Electrophysiology Laboratory by using a hand-powered rotating mechanical dilator sheaths marketed as the Evolution ${ }^{(\mathrm{r})}$ (Cook Medical) and the TightRail $^{\mathrm{TM}}$ (Spectranetics Corp.) in between July 2009 and June 2018. Patients in whom the TLE has been performed by manual traction or with a locking stylet were excluded from the study. The indications for TLE were based on the recent European Heart Rhythm Association (EHRA) and Heart Rhythm Society (HRS) recommendations ${ }^{1}$.

The study population was categorized into two as; the Evolution ${ }^{(\mathrm{r})}$ group and the TightRail ${ }^{\mathrm{TM}}$ group. First- $_{\text {- }}$ generation Evolution $^{(\mathrm{r})}$ mechanical dilator sheath was used between July 2009 and September 2014 and TightRail $^{\text {TM }}$ mechanical dilator sheath was used between September 2014 and June 2018 because of the availability and reimbursement policy of National Social Security System. There is no patient cross-over between the two groups. Laser-assisted sheaths or the second-generation Evolution ${ }^{\mathrm{R} / \mathrm{L}}$ mechanical dilator sheath were unavailable and not re-imbursed during the study period in our country. The study data were collected by using electronic medical records, files, and National Death Reporting System. The study complied with the principles outlined in the Declaration of Helsinki and approved by our local institutional ethics committee.

\section{Lead extraction technique}

The variable technical properties of both mechanical dilator sheaths were defined in Supplementary File 1 . The TLE procedure was performed in the Electrophysiology Laboratory under deep sedation and local anesthesia with invasive blood pressure monitoring via femoral or radial route, non-invasive oxygen saturation monitoring, and a cardiothoracic surgery team standby. A thorough evaluation of pacemaker (PM/ICD) was performed before the intervention, including the assessment of the degree of pacemaker dependency and 
temporary transvenous pacing was established if necessary. After the skin preparation, the generator pocket was opened, and the device generator was disconnected from the leads. The leads were separated from the scar tissue by blunt dissection. Simple manual traction via standard stylet was initially attempted. If manual traction was not successful, a systematic approach using locking stylet (Liberator Universal Locking Stylet in Evolution ${ }^{(\mathrm{r})}$ group, Cook Medical) (Lead Locking Device EZ in the TightRail ${ }^{\mathrm{TM}}$ group, Spectranetics Corp.) for TLE. If this systematic approach was unsuccessful, mechanical dilator sheaths were used for both atrial, right ventricular, and coronary sinus leads.

Mechanical dilator sheath was then positioned over the targeted lead. The operator pulls the handle of the dilator sheath, which causes rotation of the cutting tip. The dilator sheath moves along the lead body by cutting fibrous adhesions via the distal metal tip or blade. In the Evolution ${ }^{(\mathrm{r})}$ mechanical dilator sheath system, the outer polymer sheath covers the distal tip while advancing over the lead in the tracts free from adherences to protect the venous wall from damage and when fibrous attachments met, the cutting tip uncovered from the outer sheath. In the TightRail ${ }^{\mathrm{TM}}$ mechanical dilator sheath system, the shielded blade dilates the fibrous attachments by rotating $270 \mathrm{deg}$ clockwise and 270degcounterclockwise with each full trigger activation while extending the blade just $0.5 \mathrm{~mm}$. Once the fibrous attachments are cut, the outer sheath is advanced until another area of attachment is encountered. After the release of leads from fibrous tissue, the leads were pulled back into the sheath and removed. In case of failure with an antegrade approach and presence of free-floating lead remnants, a femoral or jugular approach with Multisnare (Multi-Snare, PFM, Koln, Germany) was used to grasp the remaining part and to complete the procedure. For patients requiring replacement of their lead, a new lead system was implanted through the same vein in case of lead malfunction or upgrade to new technology. In the case of device infection, the subclavian vein on the opposite side was used after the eradication of infectious microorganisms according to the recommendations of the recent guidelines ${ }^{1}$. In PM-dependent patients, re-implantation was performed in the same session if the extraction was due to non-infectious causes. In PM-dependent patients with cardiac device infection, a temporary PM was implanted through the contralateral jugular vein.

During the first $48 \mathrm{~h}$ after the procedure, continuous non-invasive blood pressure, oxygen saturation, and electrocardiographic monitoring were made and echocardiographic evaluation just after the intervention and before discharge was performed. At each follow-up visit, a thorough device interrogation was added to the patient assessment with clinical evaluation, electrocardiography, chest X-ray, and transthoracic echocardiography when necessary.

\section{Study Outcomes}

Procedural/clinical success, failure, and complications

The success of TLE was determined through complete procedural and clinical criteria. Complete procedural success was defined as the removal of all targeted leads and lead material from the vascular space without permanently disabling complication or procedure-related mortality. Clinical success was defined as the removal of all targeted leads and lead material from the vascular space or retention of a small portion of the lead $(<4 \mathrm{~cm})$ that does not negatively impact the outcome goals of the procedure. This might be the tip of the lead or a small part of the lead when the remaining portion does not increase the risk of perforation, embolic events, the perpetuation of infection or cause any undesired outcome. "Failure" was defined as the inability to achieve either complete procedural or clinical success or the development of any permanently disabling complication or procedural-related death. Complications were defined as major orminor, according to previously published guidelines ${ }^{1}$.

\section{Survival outcomes}

All-cause mortality was defined as the mortality from all causes of death for our study population during follow-up period. Mortality data was recorded for each patient from National Death Recording System.

\section{Statistical analysis}

Statistical analysis was performed using the NCSS (Number Cruncher Statistical System) 2007 (Kaysville, 
Utah, USA). Continuous variables were presented as mean \pm standard deviation and median with interquartile ranges as appropriate and categorical variables as frequency and percentage. To test the normality of distribution, the Kolmogorov Smirnov test was used. Differences between the two groups were evaluated using Student's $t$-test for normally distributed variables and Mann-Whitney $U$ test for variables without normal distribution. The Chi-square or Fisher's exact test was used to compare categorical variables as appropriate. The Wilcoxon Signed Ranks Test was used in the evaluation of hemoglobin measurements before and after the procedure. Survival analysis with a log-rank test was conducted for the combined end-point of death between the two groups, and Kaplan-Meier curves were created. Logistic regression analysis was used to evaluate the independent effects of the baseline variables on procedure failure and mortality. The odds ratios (OR) and $95 \%$ confidence intervals (CI) were calculated. A p-value $<0.05$ was considered significant.

\section{RESULTS}

Between July 2009 and June 2018, a total of 566 endovascular leads were extracted from 302 patients [Evolution ${ }^{\circledR}$ group (133 patients with 233 leads) and TightRail ${ }^{\text {TM }}$ group (169 patients with 333 leads)]. The baseline demographic, clinical and laboratory data of the study groups were represented in Table 1. Diabetes mellitus, hypertension, coronary artery disease, and HFrEF were more prevalent in the TightRail ${ }^{\mathrm{TM}}$ group $(\mathrm{p}<0.05)$.

The details of CIEDs and TLE procedure were shown in Table 2. The mean number of extracted leads per patient was $1.8 \pm 0.73$, and the median lead dwell time was 5.0 (0.6-33) years. All ICD leads were of dual coils, and all coronary sinus electrodes have a passive fixation mechanism. Re-implantation was performed in 216 $(71.5 \%)$ patients during the index TLE procedure. Leads with active fixation mechanism and coronary sinus leads were more common in the TightRail ${ }^{\mathrm{TM}}$ group $(\mathrm{p}<0.05)$. The most common TLE indications in the Evolution ${ }^{\circledR}$ and TightRail ${ }^{\mathrm{TM}}$ groups were lead malfunction $(57.9 \%)$ and CIED-related infection $(49.1 \%)$, respectively $(\mathrm{p}<0.05)$. Among different sheath sizes, $13 \mathrm{~F}$ in the TightRail ${ }^{\mathrm{TM}}$ group and $9 \mathrm{~F}$ in the Evolution ${ }^{\circledR}$ group were more frequently used $(\mathrm{p}<0.05)$.

Complete procedural and clinical success were achieved in 259 (85.8\%) and 270 (89.4\%) patients using mechanical dilator sheaths alone, respectively. There was no statistically significant difference between Evolution $\mathbb{R}$. and TightRail ${ }^{\mathrm{TM}}$ groups in complete procedural ( $86.9 \%$ vs. $\left.84.2 \%, \mathrm{p}>0.05\right)$ and clinical success $(90.6 \%$ vs. $80 \%, \mathrm{p}>0.05$ ) (Figure 1). Rescue snaring system was used because of lead fracture in 29 patients $(9.6 \%$ ) (11.2\% in Evolution_ ( vs. $8.2 \%$ in TightRail ${ }^{\mathrm{TM}}$ group, $\left.\mathrm{p}=0.286\right)$. Totally, clinical success was achieved in 298 patients $(98.6 \%)$ using both mechanical dilator sheath and snaring system $\left(99.2 \%\right.$ for Evolution ${ }^{\circledR}$ group vs. $98 \%$ for TightRail ${ }^{\mathrm{TM}}$ group). Rescue snaring system was only failed in one patient in the TightRail ${ }^{\mathrm{TM}}$ group due to trapping of 3 electrodes in the right femoral vein that were extracted surgically. Three patients failed with mechanical dilator sheaths without using rescue snaring system ( $\mathrm{n}=2$ in TightRail ${ }^{\mathrm{TM}}$ group vs. $\mathrm{n}=1$ in Evolution ${ }^{\circledR}$ group). Among 2 patients in the TightRail ${ }^{\mathrm{TM}}$ group, the procedure was terminated in one patient due to the loss of consciousness during TLE in whom intracranial hemorrhage and subsequent death was observed, and in another patient the electrode was left in the right ventricle due to failure with rescue tools. In one patient in the Evolution ${ }^{\circledR}$ group, a major vascular injury that required surgical intervention developed, and the one electrode was extracted completely by surgically. Major and minor complications were observed in $2.3 \%(\mathrm{n}=7)$ and $9.3 \%(28)$ of patients which were similar among study groups ( $\mathrm{p}>0.05)$ (Figure $2 \& 3$ ). Efficacy and safety outcome data of the study groups were shown in Table 3.

At median 36.6 (0.2-117.5) months follow-up duration [74.6 (0.6-117.5) months for Evolution ${ }^{\circledR}$ group vs. $25.8(0.2-63.5)$ months for TightRail ${ }^{\mathrm{TM}}$ group, $\left.\mathrm{p}=0.001\right]$, all-cause mortality was observed in 73 patients (25.6\% in Evolution ${ }^{\circledR}$ group vs. 23.1 in TightRail ${ }^{\mathrm{TM}}$ group, $\mathrm{p}=0.616$ ). When the survival curves of both groups are evaluated with log-rank test, there is a statistically significant difference between Evolution ${ }^{\circledR}$ and TightRail ${ }^{\mathrm{TM}}$ groups; because of the survival duration of Evolution ${ }^{\circledR}$ group was more prolonged than TightRail $^{\mathrm{TM}}$ group due to that Evolution ${ }^{\circledR}$ group had longer follow-up $(\mathrm{p}<0.001)$ (Figure 4)

Baseline demographic, clinical, laboratory and procedural characteristics of patients according to the procedural failure and all-cause mortality were shown in Supplementary File 2 and 3, respectively . In multivariable 
logistic regression analysis, the presence of HFrEF (OR: 5.73, 95\% C.I.: 1.49-22.0, $\mathrm{p}=0.011$ ), increased baseline aPTT level (OR: 1.09, 95\% C.I.: 1.03-1.17, $\mathrm{p}=0.006)$ and baseline GFR level $<60 \mathrm{ml} / \mathrm{min} / 1.73 \mathrm{~m}^{2}$ (OR: 2.81, 95\% C.I.: $1.12-7.08, \mathrm{p}=0.028)$ were associated with a higher risk of all-cause mortality. Furthermore, baseline leukocyte count (OR: 1.20, 95\% C.I.: 1.02-1.42, $\mathrm{p}=0.028$ ), number of extracted leads (OR: 2.91, 95\% C.I.: 1.45-5.86, $\mathrm{p}=0.003$ ) and lead dwell time (OR: 1.08, 95\% C.I.: 1.003-1.162, $\mathrm{p}=0.041$ ) were found as independent predictors of TLE procedural failure.

\section{DISCUSSION}

The major findings of our single-center large-scale cohort study were as follows; (1) the TLE using either Evolution ${ }^{\circledR}$ or TightRail ${ }^{\mathrm{TM}}$ hand-powered rotational mechanical dilator sheaths was associated with high efficacy and acceptable complication rates. Both procedural/clinical success and major/minor complication rates were also similar between Evolution ${ }^{\circledR}$ or TightRail ${ }^{\mathrm{TM}}$ groups. Additionally, there was no difference in all-cause mortality rates at long-term follow-up between each group. The presence of heart failure, baseline coagulopathy and impaired renal functions were associated with an increased risk of all-cause mortality.

Among various available TLE tools, hand-powered rotating mechanical dilator sheaths are preferred by many operators during extraction of chronically implanted leads with fibrotic adhesions to the vascular and/or endocardial surfaces ${ }^{8,9}$. In our study, the TLE has been performed in patients with median lead dwell time of five years by using either first-generation Evolution ${ }^{\circledR}$ or TightRail ${ }^{\text {TM }}$ systems at different time intervals because of the availability and governmental re-imbursement policy. Evolution ${ }^{\circledR}$ system has been associated with excellent clinical and procedural success with low complication rates in previous studies 9-11. In our study, complete clinical success and procedural successes were $99.2 \%$ and $94.0 \%$, respectively, as in other studies, complete clinical and procedural success rates have been mostly $>95 \%{ }^{8,11}$. The clinical and procedural success rates were $88.0 \%$ and $84.2 \%$ without using rescue methods in our study. In previous studies, complete success rate without using rescue methods and tools was in the range of $69 \%-94.5 \%$, and these studies major and minor complication rate was reported as $0 \%-4.2 \%$ and $0 \%-12 \%$, respectively $4,6,10$. In our Evolution ${ }^{\circledR}$ group, major and minor complication rates were $3.8 \%$ and $10.5 \%$, respectively, with compatible low complication rates as studies in the literature. The data about the TightRail ${ }^{\mathrm{TM}}$ system is limited in the literature. In a small observational study, the complete procedural and clinical success rates without rescue methods were $96 \%$ and $100 \%$, respectively ${ }^{5}$. In a larger retrospective multi-center study, chronically implanted 147 leads in 100 patients were extracted by using TightRail ${ }^{\mathrm{TM}}$ mechanical dilator sheath; complete success rate was $91 \%$, and major complication rate was $2 \%^{12}$. In our study, the complete procedural and clinical success rates were $86.9 \%$ and $90.5 \%$ using TightRail $^{\mathrm{TM}_{\text {system }} \text { without }}$ rescue methods. Complete clinical success was increased to $98.2 \%$ with use of rescue snaring system. Major and minor complication rates were $1.2 \%$ and $8.2 \%$, respectively, in accordance with studies in the literature.

In the literature, the need for rescue tools during TLE with mechanical dilator sheaths was highly variable (range of 2.4-27.1\%) due to used technique, extraction tool, patient and lead characteristics ${ }^{7,9,13}$. In our study, the need for rescue tools was observed in $11.2 \%$ of Evolution ${ }^{\circledR}$ group and $8.2 \%$ of TightRail ${ }^{\mathrm{TM}}$ group $(\mathrm{p}>0.05)$. Higher lead dwell time and lead burden were associated with an increased need for rescue tools and major complication rates ${ }^{14}$. TLE indications of lead dysfunction and infection were also more prevalent in patients with lead breakage, incomplete lead extraction, and need for rescue snaring tools ${ }^{9}$. In our study, the lead dwell time, lead burden, leads with passive fixation, and infection parameters were significantly higher in patients with procedural failure who required rescue snaring tool.

In our study, major complications were totally observed in 7 patients (2.3\%). Additionally, procedure-related death was seen in 2 patients $(0.66 \%)$ which was due to intracranial hemorrhage $(n=1)$ in the TightRail ${ }^{\mathrm{TM}}$ group and haemothorax $(\mathrm{n}=1)$ in the Evolution ${ }^{\circledR}$ group. Besides these deaths, major complications included cardiac tamponade in one patient at the TightRail ${ }^{\mathrm{TM}}$ group, major vascular lacerations in 3 patients by $0.99 \%$ at Evolution ${ }^{\circledR}$ group, a cerebrovascular accident by $0.33 \%$ in Evolution ${ }^{\circledR}$ group. In the ELECTRa registry, procedure-related death was seen in the range of $0.3-0.8 \%$ rates, and our study data is similar to this registry. In the ELECTRa registry, the cerebrovascular accident was followed by $0.06 \%$, and vascular laceration was followed by $0.4-0.9 \%$, and our data was similar to this registry ${ }^{8}$. Patients with cardiac 
tamponade had both atrial and ventricular PM electrodes with passive fixation mechanism in which lead dwell time was ten years. The myocardial avulsion was thought to be during extraction of the atrial electrode which was successfully repaired surgically. In previous studies, massive pericardial effusion was reported by $0.25-0.59 \%$ which was $0.33 \%$ in our study accordingly. In the ELECTRa registry analysis, $84.5 \%$ of major complications were cardiovascular avulsion, and cardiac avulsion with tamponade is the most common major complication by $61.2 \%{ }^{15}$. In our study, $71 \%$ of major complications were cardiovascular complications, and cardiac avulsion was observed in two patients by $28.5 \%$. In the ELECTRa registry, the minor complication rate was reported as $5 \%$ (range 4.3-5.7\%) which was $9.2 \%$ in our study. The majority of minor complications in our study was pocket hematoma by $60 \%$, but the drainage was only required in $2(0.6 \%)$ of the patients In previous studies, the rate of hematoma requiring drainage was in the range of $0.9-1.6 \%^{16,17}$. In our study, the minor complication rate was higher than the ELECTRa registry, and because of mostly hematoma, which didn't require drainage, this condition did not cause any clinical problems.

There was no difference between TightRail ${ }^{\mathrm{TM}}$ and Evolution ${ }^{\circledR}$ groups in regard to all-cause mortality at long-term follow-up in our study. To the best of our knowledge, our study is unique with its largest sample size of TightRail ${ }^{\mathrm{TM}}$ group in the literature in addition to long-term mortality outcomes of both device technology. Higher leukocyte count, lead burden, and lead dwell time were associated with a higher risk of procedural failure; and presence of heart failure, coagulopathy and chronic renal disease were associated with a higher risk of all-cause mortality in our study. In previous studies, it was observed that the lead burden associated with a 3.5 fold increased risk of any complication. Moreover, TLE for infection, and high CRP levels were found to be associated with all-cause mortality ${ }^{18,19}$. In a large-scale study with extracted 5521 leads that evaluated risk factors of procedural failure, major complications, and all-cause mortality, low platelet counts and higher INR levels $(>1.2)$ were associated with major complications and 30-day death ${ }^{20}$. Furthermore, heart failure and renal dysfunction increased the 30-day mortality by 1.3-8.5 and 4.8 fold, respectively ${ }^{21,22}$.

Our study results have important clinical implications. The TLE using either TightRail ${ }^{\text {TM }}$ or Evolution ${ }^{\circledR}$ mechanical dilator sheaths can be performed with an excellent clinical/procedural success and acceptable complication rates. These findings suggest that these extraction tools require a learning curve and should be used by experienced operators with a cardiothoracic surgery team on standby to cope with any complication.

Our study results should be interpreted with some limitations. First, the study groups were non-randomized and the data was collected retrospectively in this cohort study. Second, the impact of learning curve on outcomes of Evolution ${ }^{\circledR}$ group which was available at first could not be ignored. Third, data for procedure and fluoroscopy time were not available for all participants. Fourth, second generation Evolution ${ }^{\mathrm{R} / \mathrm{L}}$ mechanical dilator sheath couldn't be used during the study period. At last, the availability of TLE devices mainly depends on the re-imbursement policy of our National Social Security System which limits the randomization. Furthermore, non-rotational dissection tools and laser sheath were not available in our country.

In conclusion, our results showed that the TLE by using either TightRail ${ }^{\mathrm{TM}}$ or Evolution ${ }^{\circledR}$ hand-powered rotational mechanical dilator sheath systems was highly effective with acceptable safety results. However, rescue extraction tools and backup cardiothoracic surgery support should be available on site. The selection of TLE tool should be based on the operator preferance/experience, availability and re-imbursement of the devices. Continued investigation is required to compare safety, success, and complication rates with other techniques.

\section{REFERENCES}

1. Bongiorni MG, Burri H, Deharo JC, et al. 2018 EHRA expert consensus statement on lead extraction: recommendations on definitions, endpoints, research trial design, and data collection requirements for clinical scientific studies and registries: endorsed by APHRS/HRS/LAHRS.Europace. 2018;20(7):1217.

2. Maytin M, Jones SO, Epstein LM. Long-term mortality after transvenous lead extraction. Circulation: Arrhythmia and Electrophysiology.2012;5(2):252-257. 
3. Oto A, Aytemir K, Yorgun H, et al. Percutaneous extraction of cardiac pacemaker and implantable cardioverter defibrillator leads with evolution mechanical dilator sheath: a single-centre experience.Europace. 2010;13(4):543-547.

4. Witte OA, Adiyaman A, Smit JJJ, et al. Success and complication rates of lead extraction with the first-vs. the second-generation Evolution mechanical sheath. Ep Europace. 2016;19(10):1717-1722.

5. Aytemir K, Yorgun H, Canpolat U, et al. Initial experience with the TightRail Rotating Mechanical Dilator Sheath for transvenous lead extraction. Europace. 2016;18(7):1043-1048.

6. Delnoy PPH, Witte OA, Adiyaman A, et al. Lead extractions: the Zwolle experience with the Evolution mechanical sheath. Ep Europace.2015;18(5):762-766.

7. Cay S, Ozeke O, Ozcan F, Topaloglu S, Aras D. Comparison of two types of rotational mechanical dilatator sheath: Evolution((R)) and TightRail(). Pacing Clin Electrophysiol. 2019;42(9):1226-1235.

8. Bongiorni MG, Kennergren C, Butter C, et al. The European lead extraction ConTRolled (ELECTRa) study: a European heart rhythm association (EHRA) registry of transvenous lead extraction outcomes.European heart journal. 2017;38(40):2995-3005.

9. Sharma S, Ekeruo IA, Nand NP, et al. Safety and Efficacy of Transvenous Lead Extraction Utilizing the Evolution Mechanical Lead Extraction System: A Single-Center Experience. JACC Clin Electrophysiol. 2018;4(2):212-220.

10. Mazzone P, Tsiachris D, Marzi A, et al. Advanced techniques for chronic lead extraction: heading from the laser towards the evolution system. Europace. 2013;15(12):1771-1776.

11. Starck CT, Gonzalez E, Al-Razzo O, et al. Results of the Patient-Related Outcomes of Mechanical lead Extraction Techniques (PROMET) study: a multicentre retrospective study on advanced mechanical lead extraction techniques. Europace. 2020.

12. Love C, Kress DC, Al-Ahmad A, Gokoglan Y, Canby R. 16-64: multi-center experience with the spectranetics tightrail lead extraction system. 2016.

13. Domenichini G, Gonna H, Sharma R, et al. Non-laser percutaneous extraction of pacemaker and defibrillation leads: a decade of progress.Europace. 2017;19(9):1521-1526.

14. Gould J, Sidhu BS, Porter B, et al. Prolonged lead dwell time and lead burden predict bailout transfemoral lead extraction. Pacing Clin Electrophysiol. 2019;42(10):1355-1364.

15. Zucchelli G, Di Cori A, Segreti L, et al. Major cardiac and vascular complications after transvenous lead extraction: acute outcome and predictive factors from the ESC-EHRA ELECTRa (European Lead Extraction ConTRolled) registry. EP Europace. 2018;21(5):771-780.

16. Brunner MP, Cronin EM, Jacob J, et al. Transvenous extraction of implantable cardioverter-defibrillator leads under advisory - a comparison of Riata, Sprint Fidelis, and non-recalled implantable cardioverterdefibrillator leads. Heart Rhythm.2013;10(10):1444-1450.

17. Ji SY, Gundewar S, Palma EC. Subclavian venoplasty may reduce implant times and implant failures in the era of increasing device upgrades. Pacing and Clinical Electrophysiology.2012;35(4):444-448.

18. Agarwal SK, Kamireddy S, Nemec J, Voigt A, Saba S. Predictors of complications of endovascular chronic lead extractions from pacemakers and defibrillators: A single-operator experience. Journal of cardiovascular electrophysiology. 2009;20(2):171-175.

19. Hamid S, Arujuna A, Ginks M, et al. Pacemaker and defibrillator lead extraction: Predictors of mortality during follow-up. Pacing and clinical electrophysiology. 2010;33(2):209-216. 
20. Brunner MP, Cronin EM, Duarte VE, et al. Clinical predictors of adverse patient outcomes in an experience of more than 5000 chronic endovascular pacemaker and defibrillator lead extractions. Heart Rhythm. 2014;11(5):799-805.

21. Wazni O, Epstein LM, Carrillo RG, et al. Lead extraction in the contemporary setting: the LExICon study: an observational retrospective study of consecutive laser lead extractions. Journal of the American College of Cardiology. 2010;55(6):579-586.

22. Byrd CL, Wilkoff BL, Love CJ, et al. Intravascular extraction of problematic or infected permanent pacemaker leads: 1994-1996.Pacing and Clinical Electrophysiology. 1999;22(9):1348-1357.

\section{Figure Legends}

Figure 1 . The comparison of procedural success and failure rates without using rescue tools.

Figure 2 . The distribution and comparison of major complications according to the mechanical dilator sheath types.

Figure 3 . The distribution and comparison of minor complications according to the mechanical dilator sheath types.

Figure 4 . Kaplan-Meier curve illustrating the cum suvival of $\operatorname{TightRail}^{\mathrm{TM}}$ and Evolution ${ }^{(\mathrm{r})}$ groups during long-term follow-up ( $<<0.001$, log-rank).
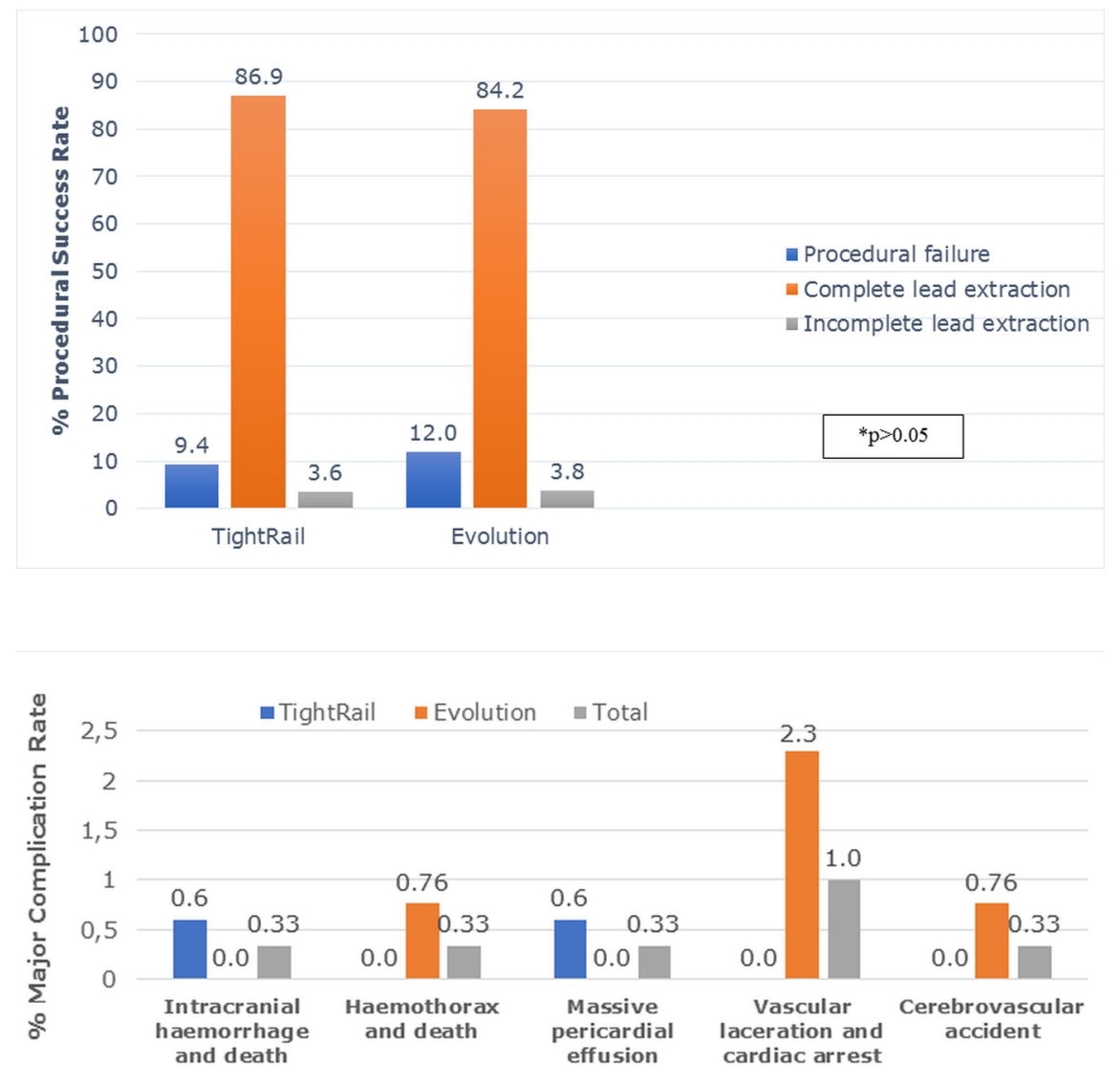

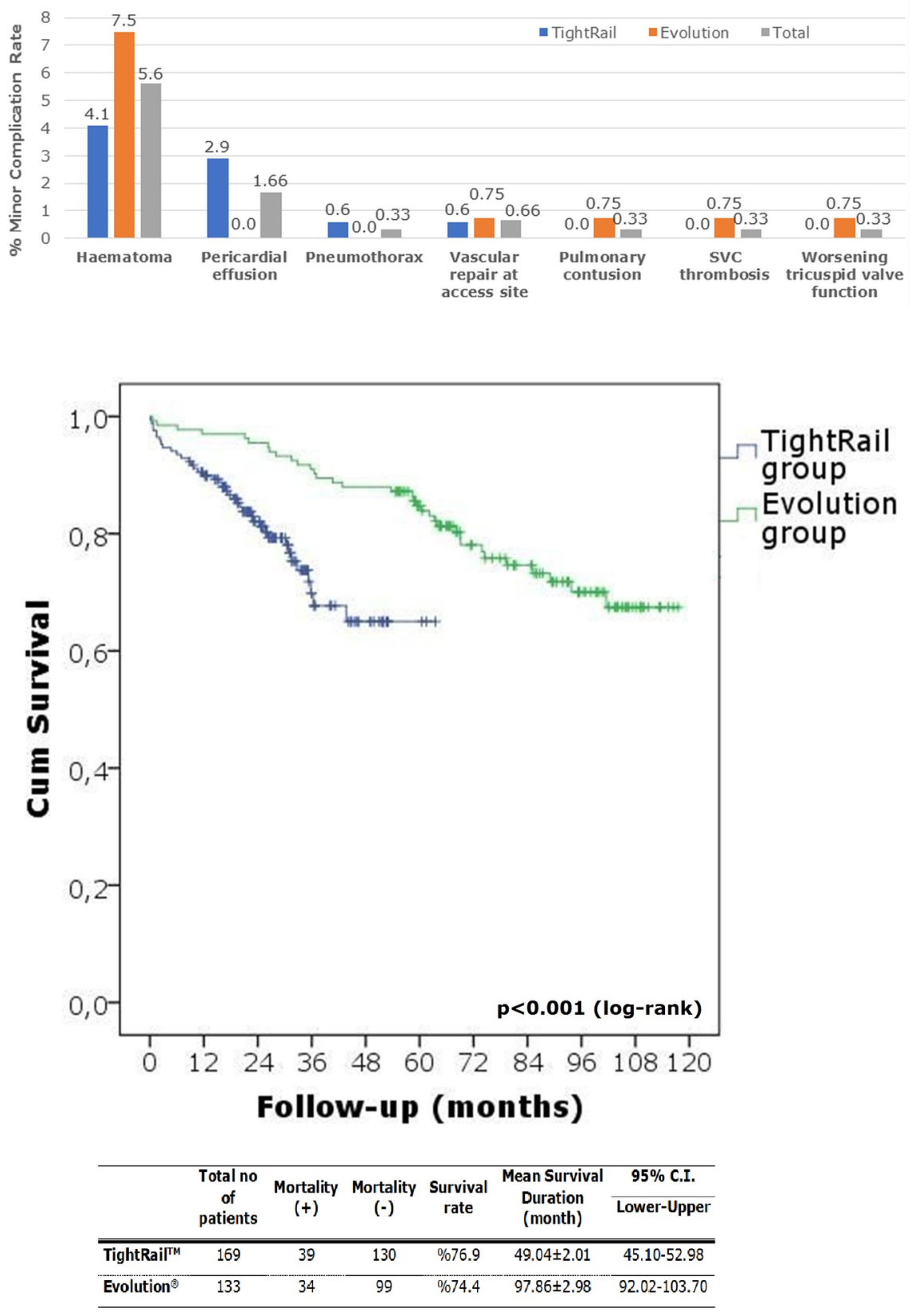

\section{Hosted file}

Table 1.docx available at https://authorea.com/users/335152/articles/461029-comparisonof-acute-and-long-term-outcomes-of-evolution-and-tightrail-mechanical-dilator-sheathsduring-transvenous-lead-extraction

\section{Hosted file}

Table 2.docx available at https://authorea.com/users/335152/articles/461029-comparisonof-acute-and-long-term-outcomes-of-evolution-and-tightrail-mechanical-dilator-sheathsduring-transvenous-lead-extraction 


\section{Hosted file}

Table 3.docx available at https://authorea.com/users/335152/articles/461029-comparisonof-acute-and-long-term-outcomes-of-evolution-and-tightrail-mechanical-dilator-sheathsduring-transvenous-lead-extraction
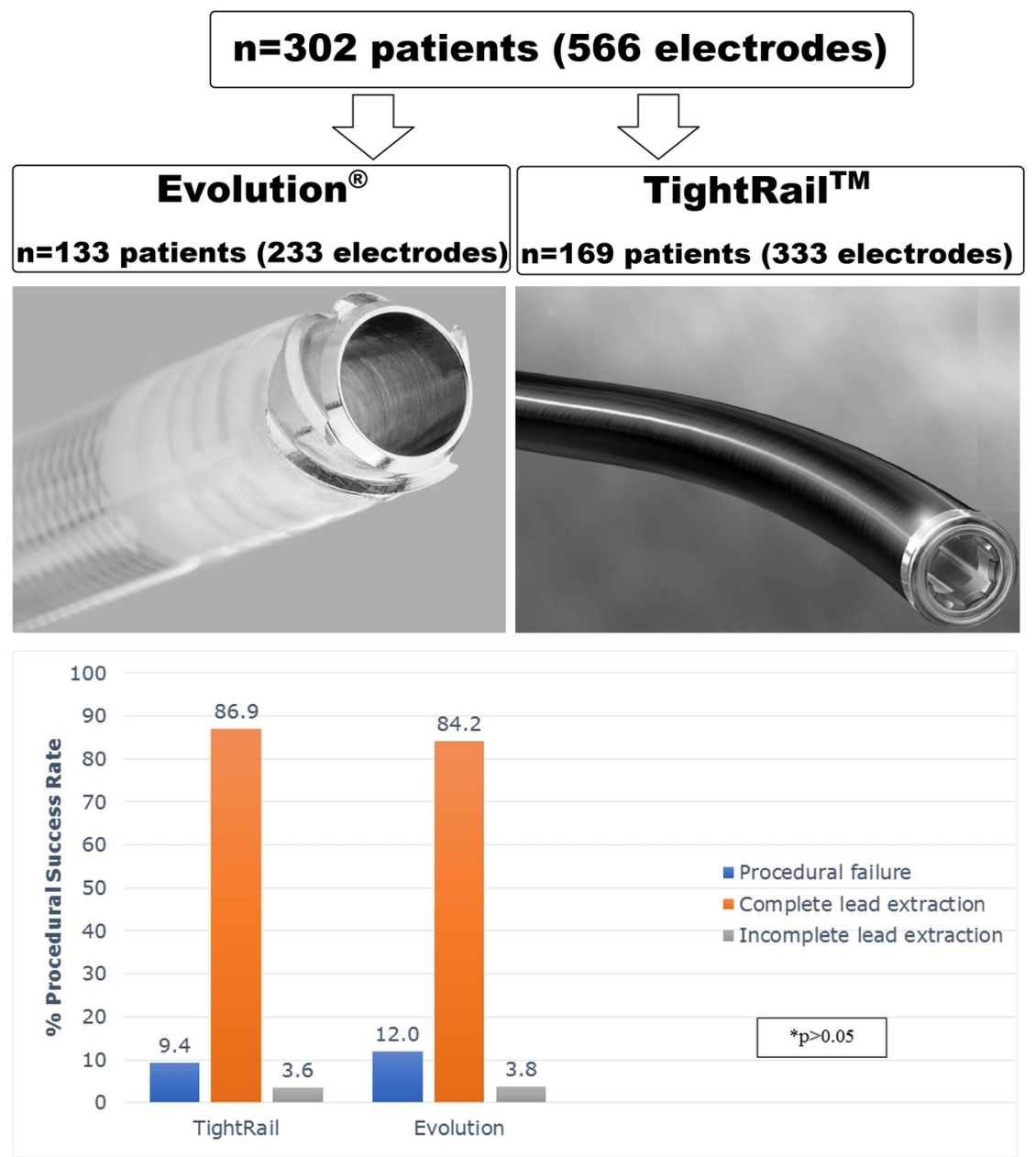